\title{
Análise geo-espacial de malária no município de Cametá - PA, no período de 2008 a 2018
}

\author{
Lucas Henrique da Silva e Silva \\ Mestrando do Programa de Pós-Graduação em Biologia Parasitária na Amazônia (UEPA) \\ $\triangle$ lucashenriqueuepa@gmail.com \\ Rammon David Estumano Cohen \\ Licenciado em Ciências Naturais - Habilitação em Biologia (UEPA) \\ Beatriz Oliveira Miranda \\ Mestranda do Programa de Pós-Graduação em Saúde Animal na Amazônia (UEPA) \\ André Luis Pinto Furtado \\ Licenciado em Ciências Naturais - Habilitação em Biologia (UEPA) \\ Marcelo Coelho Simões \\ Mestre em Ciências Ambientais - PPGCA (UEPA) \\ Cléa Nazaré Carneiro Bichara \\ Doutora em Agentes Infecciosos e Parasitários (UEPA) \\ Jessica Herzog Viana \\ Doutora em Ciências Biológicas (UFPR), Pós-Doutoranda do \\ Programa de Pós-Graduação em Ciências Ambientais (UEPA)
}

Recebido em 7 de junho de 2020

Aceito em 2 de julho de 2020

\begin{abstract}
Resumo:
A malária é uma doença parasitária grave, que representa um importante problema de saúde pública. O presente estudo teve como objetivo avaliar os principais aspectos epidemiológicos da malária no período de 2008 a 2018. Trata-se de um estudo descritivo - exploratório desenvolvido no município de Cametá, Pará, Brasil. As amostras foram obtidas do banco de dados SIVEP-Malária do Ministério da Saúde e a ferramenta Google Earth, para mapeamento das prováveis áreas de infecção. No ano de 2012, foram notificados 2340 casos de agravos de malária no município, representando uma redução de $83 \%$ em comparação ao ano de 2011, a incidência parasitária anual foi de 13,73 casos/1000 habitantes em 2011 e 2,34 casos/1000 habitantes no ano de 2012, representando uma redução de $83 \%$. No entanto, houve um aumento de 68,4\% no ano de 2018 em relação ao ano de 2017. Identificou-se que a espécie de Plasmodium predominante foi o P. vivax com 23.723 casos, seguido do P. falciparum com 86 casos e um caso com o P. malariae. Observou-se que 418 casos foram confirmados no teste rápido para alguma espécie parasitária que não seja $P$. falciparum e 15 casos houve a presença de duas espécies de Plasmodium. O georeferenciamento permitiu observar áreas de prováveis infecções na zona urbana do município, bem como nas áreas rurais do distrito. É de suma importância a descentralização do sistema de saúde, para que as medidas de tratamento, principalmente os medicamentos antimaláricos, e prevenção estejam ao alcance dos indivíduos que vivem em áreas de risco da infecção.

Palavras-chave: Amazônia, Plasmodium falciparum, Plasmodium vivax, Infecto-parasitária.
\end{abstract}




\title{
Geospatial analysis of malaria in the municipality of Cametá - PA, from 2008 to 2018
}

\begin{abstract}
:
Malaria is a serious parasitic disease that represents a major public health problem. This study aimed to evaluate the main epidemiological aspects of malaria from 2008 to 2018. This is a descriptive exploratory study developed in the city of Cametá, Pará, Brazil. Samples were obtained from the Ministry of Health's SIVEP-Malaria database and the Google Earth tool to map likely areas of infection. In the year 2012, 2340 cases of malaria were reported in the municipality, representing a reduction of $83 \%$ compared to 2011, the annual parasitic incidence was 13.73 cases / 1000 inhabitants in 2011 and 2.34 cases / 1000 inhabitants in 2012, representing a reduction of $83 \%$. However, there was an increase of $68.4 \%$ in 2018 compared to 2017. It was found that the predominant Plasmodium species was P. vivax with 23,723 cases, followed by P. falciparum with 86 cases and 1 case with P. malariae. It was observed that 418 cases were confirmed in the rapid test for any parasitic species other than P. falciparum and 15 cases had two Plasmodium species. The georeferencing allowed to observe areas of probable infections in the urban area of the municipality, as well as in the rural areas of the district. In view of the affected areas, decentralization of the health system is of paramount importance so that treatment measures, especially antimalarial drugs, and prevention are within the reach of individuals living in areas at risk of infection.
\end{abstract}

Keywords: Amazon, Plasmodium falciparum, Plasmodium vivax, Infect parasitic.

\section{Análisis geoespacial de la malaria en el municipio de Cametá - PA, de 2008 a 2018}

\section{Resumen:}

La malaria es una grave enfermedad parasitaria que representa un importante problema de salud pública. Este estudio tuvo como objetivo evaluar los principales aspectos epidemiológicos de la malaria de 2008 a 2018. Este es un estudio exploratorio descriptivo desarrollado en la ciudad de Cametá, Pará, Brasil. Se obtuvieron muestras de la base de datos SIVEP-Malaria del Ministerio de Salud y la herramienta Google Earth para mapear zonas de probables infección. En el anõ 2012, se informaron 2340 casos de malaria en el municipio, lo que representa una reducción del $83 \%$ en comparación con 2011, la incidencia parasitaria anual fue de 13.73 casos / 1000 habitantes en 2011 y 2.34 casos / 1000 habitantes en 2012, lo que representa una reducción del 83\%. Sin embargo, hubo un aumento del $68.4 \%$ en 2018 en comparación con 2017. Se identificó que la especie de Plasmodium predominante fue $P$. vivax con 23,723 casos, seguido de $P$. falciparum con 86 casos y 1 caso con $P$. malariae. Se observó que 418 casos fueron confirmados en la prueba rápida para cualquier especie parasitaria que no sea P. falciparum y 15 casos tenían dos especies de Plasmodium. La georreferenciación permitió observar zonas de probables infecciones en la zona urbana del municipio, así como en la zonas rurales del distrito. La descentralización del sistema de salud es de suma importancia para que las medidas de tratamiento, especialmente los medicamentos antipalúdicos, y la prevención estén al alcance de las personas que viven en zonas con riesgo de infección.

Palabras clave: Amazon, Plasmodium falciparum, Plasmodium vivax, Infectar parásito.

\section{INTRODUÇÃO}

O território brasileiro corresponde a aproximadamente 8,5 milhões de quilômetros quadrados e uma população que ultrapassa os 200 milhões de habitantes, com grande parte do território dentro da zona tropical, os quais quase 60\% estão na região amazônica (IBGE, 
2016). Com uma fauna e flora altamente diversificada, existe uma evidente vulnerabilidade frente às infecções vetoriais, tais como zika vírus, chikungunya, febre amarela, dengue e malária (SIQUEIRA, 2016).

Atualmente, houve grandes retrocessos no combate e tratamento de infecções por arbovirus emergentes no país, porém, se tratando especificamente da malária, houve uma queda significativa no número de casos dos últimos 35 anos, com 143 mil casos notificados no ano de 2015 por exemplo, o que corresponde em redução de $89 \%$ dos óbitos em relação ao ano de 2000 (BRASIL, 2017). Esses resultados positivos são em parte consequências dos programas que visam medidas de controle e prevenção, promovidos pelo Ministério da Saúde.

Entre 2000 e 2015, a incidência da infecção por malária entre as populações em situação de risco (casos novos) caiu para 37\% em todo o globo, nesse mesmo período, houve a diminuição da taxa de mortalidade pela doença entre as populações em risco diminuindo 60\% entre todas as faixas etárias e 65\% entre crianças menores de cinco anos (WHO, 2016), sendo essa mudança pode ser atribuída à implementação de medidas de intervenção de controle da endemia. Apesar dessa redução, a malária ainda hoje continua apresentando um impacto negativo na saúde pública e nos meios de subsistência dos indivíduos, tornando-se um agravo de notificação de saúde pública, devido a alta taxa de mortalidade nas regiões tropicais (ROGERSON et al., 2018), atingindo 212 milhões de casos da doença no ano de 2015 em todo o mundo (WHO, 2016).

A doença pode ser provocada por quatro espécies de protozoários do gênero Plasmodium, sendo elas: P. vivax, P. falciparum, P. malariae e P. ovale. No Brasil, apenas os três primeiros estão presentes, sendo o $P$. vivax e o $P$. falciparum as espécies mais recorrentes (FUNDAÇÃO OSWALDO CRUZ, 2015).

A transmissão da malária está quase que exclusivamente restrita a região amazônica (VITOR-SILVA et al., 2016), correspondendo a nove dos estados no Brasil, além de alguns casos na região extra-amazônica, como por exemplo nos estados do Espirito Santo e São Paulo (BRASIL, 2015). Segundo Brasil (2018) o estado do Pará está inserido dentro da faixa endêmica do território nacional, registrando cerca de 175.610 casos de agravo no período de 2012 a 2017.

Braz e Barcellos (2018) realizaram a distribuição dos 808 municípios da Amazônia brasileira em grupos, de acordo com os respectivos "indicadores de prioridades", onde o 
estado do Pará, por exemplo, enquadra-se em um grupo chamado "Grupo 3C", no qual busca a redução do índice de malária no estado. Neste estudo, os municípios foram classificados de acordo com os níveis de eliminação da transmissão, números de casos autóctones, Índice Parasitário Anual (IPA) e Limites Inferior e Superior, LIC e LSC respectivamente, do diagrama de corte dos índices de incidência de malária. Nesse sentido, o "Grupo 3C" classifica municípios com busca da redução da transmissão, com epidemia e com número de casos acima do LSC.

Os gestores governamentais que desenvolvem pesquisas sobre malária no país ainda possuem muitos desafios, de modo que, o Ministério da Saúde juntamente com instituições de pesquisas nacionais e internacionais devem promover estratégias e pesquisas que auxiliem no controle e combate desta endemia, a saber: ressaltar a importância dos assintomáticos em áreas de diferentes níveis de transmissão; detecção, de forma custo-efetiva de infecções subclínicas, ou mesmo o momento da transição epidemiológica se deve prever a substituição ou suspenção das intervenções de controle vetorial são algumas medidas que poderão ser tomadas (BRASIL, 2016).

Com base nisso, o presente estudo teve como objetivo avaliar os principais aspectos epidemiológicos e socioambientais da malária no município de Cametá, Estado do Pará, no período de 2008 a 2018.

\section{METODOLOGIA}

\section{Tipo de estudo}

Trata-se de um estudo do tipo ecológico, retrospectivo, descritivo, de corte transversal, de abordagem quantitativa, pois se empregou estatística descritiva para cálculos de média e frequência e posterior geração de gráficos a partir de dados secundários oficiais. 


\section{Local de estudo}

O estudo se desenvolveu no município de Cametá, Pará, Brasil, localizado na região amazônica brasileira com coordenadas geográficas 2¹3’43”s 49²9'25"O (Figura 1). 0 município possui uma extensão territorial de $3.081,367 \mathrm{~km}^{2}$, com uma temperatura média de $27^{\circ} \mathrm{C}$, taxa de precipitação de $53 \mathrm{~mm}$ nos meses mais secos e $406 \mathrm{~mm}$ no período chuvoso, com 120.896 habitantes de acordo com o último censo, sendo o oitavo município mais populoso do estado do Pará (IBGE, 2019).

Figura 1. Localização geográfica do município de Cametá, Pará, Amazônia, Brasil.

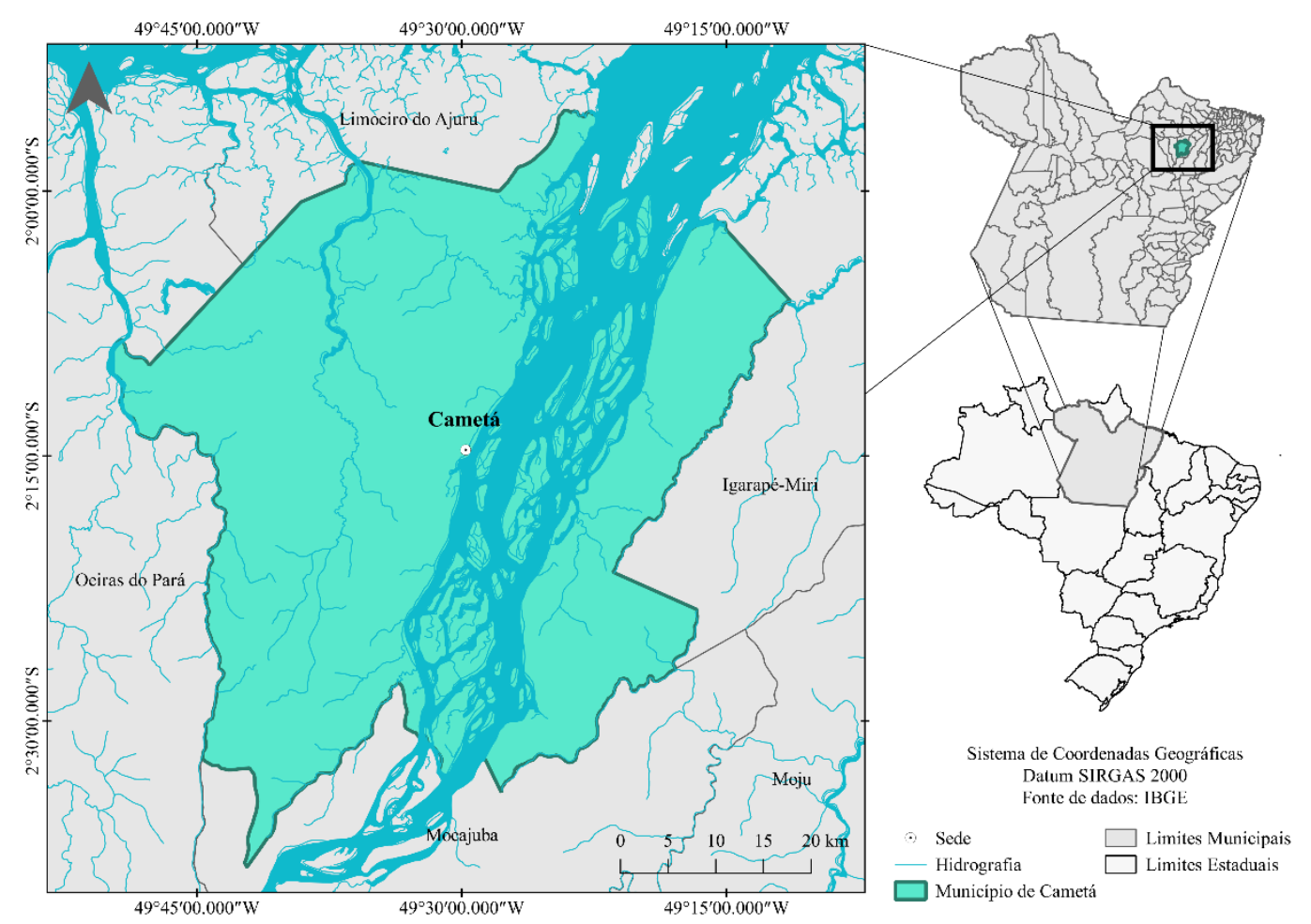

Fonte: The Weather Channel, 2019.

\section{Procedimentos de coleta de dados}

Foram utilizados dados oficiais de fontes secundárias dos arquivos do Sistema de Informação de Vigilância Epidemiológica (SIVEP Malária), da Secretaria de Vigilância em Saúde. Os dados apresentam a prevalência da malária por ano, onde se calculou o coeficiente 
de incidência, no período de 2008 a 2018. Foi aplicada estatística descritiva, cujas variáveis quali-quantitativas foram apresentadas por distribuições de frequências absolutas e relativas, utilizando a ferramenta Microsoft office Excel 2013, obtendo a frequência em porcentagem. 0 coeficiente de incidência da malária foi obtido utilizando a fórmula padrão para a obtenção do indicador, a partir do número de casos de malária: $\mathrm{CI}$ = № de casos de Malária/população total, fator de multiplicação igual a 1.000, bem como realizou-se o cálculo de incidência parasitária anual (API) (número de casos novos durante 1 ano / população x 1.000).

Ao saber que a atenção primária à saúde é fornecida gratuitamente no Brasil e que os sistemas de informação do Ministério da Saúde oferecem ampla cobertura, presume-se que os dados fornecidos pelo Departamento de Vigilância Epidemiológica da Secretaria de Vigilância Sanitária de Cametá são confiáveis.

Foram incluídos todos os dados catalogados oficialmente no banco de dados SIVEPMalária do Ministério da Saúde. Os dados não oficiais foram excluídos.

\section{Rastreio da infecção por malária}

No Brasil, sempre que um indivíduo apresenta sintomas suspeitos de malária, o mesmo é testado por agentes endêmicos qualificados que monitoram microrregiões. $O$ método padrão-ouro utilizado para o diagnóstico da malária é o esfregaço de sangue fino e espesso, que é examinado por microscopistas treinados do Sistema de Vigilância Epidemiológica do Ministério da Saúde, e posteriormente revisado por especialistas, para confirmar os resultados. As infecções foram categorizadas por espécies de parasitas: $P$. falciparum, P. vivax, P. malariae e P. ovale ou infecções mistas.

O georeferenciamento da provável área de infecção foi realizado utilizando o banco de dados SIVEP-Malária e a ferramenta Google Earth, objetivando um banco de dados de rastreamento de infecções. 


\section{RESULTADOS E DISCUSSÃo}

Ao analisar o banco de dados do Sistema de Informação de Vigilância Epidemiológica - Malária (SIVEP-Malária) identificou-se um total 47.671 casos agravos notificados da doença no período de 2008 a 2018, destacando os anos 2011 e 2014, os quais apresentam o maior número de casos da doença com 17.257 e 12.609, respectivamente, e evidenciando um aumento do número de casos a partir de 2017 (Figura 02).

Figura 2. Número de casos agravos de malária em relação ao ano de ocorrência em Cametá- PA.

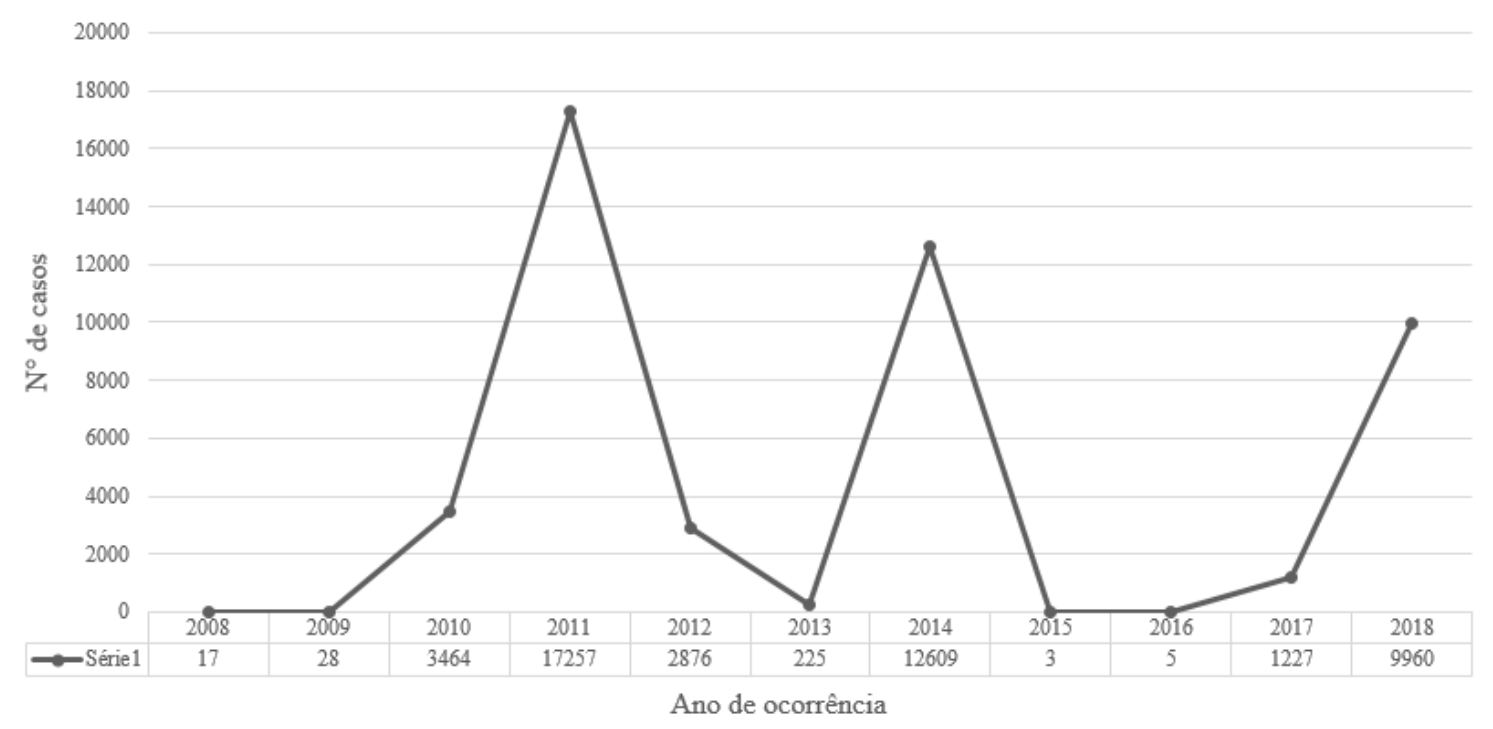

Fonte: SIVEP-Malária, 2019.

Em 2012, foram notificados 2.876 casos agravos de malária no município, representando uma redução de $83 \%$ na taxa de incidência em comparação ao ano anterior (2011), com incidência parasitária anual de 142,7 casos/1000 habitantes no ano de 2011 e reduzido à 23,8 casos/1000 habitantes em 2012. Os resultados apresentados são melhores do que os registrados por Brasil (2015) no estado do Pará, que também se destacou na região amazônica por reduzir os 115 mil casos de malária registrados no ano de 2011 para 79 mil casos em 2012, o que representa uma redução no estado de $31 \%$ em um ano.

Apesar dos 225 casos em 2013, há um crescimento alarmante de notificações em 2014 com 12.609 casos, reduzindo drasticamente nos anos seguintes, 2015 com 3 casos e 2016 com 5 casos. No entanto, em 2018 (9.960 casos) houve um aumento de 87,7\% em relação ao ano de 
2017 (1.227 casos), o qual já apresentava um aumento se comparado a 2016. O baixo número de casos da doença nos anos de 2008 e 2009, segundo Oliveira et al. (2010) podem estar relacionadas às ações do Programa Nacional de Controle da Malária (PNCM) de fortalecimento das capacidades de gestão das localidades, em vista de um aumento da rede de tratamento e diagnóstico. Entretanto há vários outros fatores que estão associados à incidência da doença, como, por exemplo, desmatamento (Recht et al., 2017), taxa de precipitação (Romi et al., 2012), precipitação dos rios (Wolfarth-Couto et al. 2019) e entre outros.

Para Cosner et al. (2009) ocorreram aumentos significativos dos problemas causados por doenças transmitidas por vetores em decorrência da urbanização, globalização, desmatamento e desenvolvimento econômico. Estudos de Achcar et al. (2011) mostram o desenvolvimento humano, o crescimento populacional, o desmatamento e as alterações ecológicas associadas ao aumento dos riscos de incidência de malária na Amazônia legal. Desse modo, pressupõem que o aumento da incidência de malária no município de Cametá pode ter relação com tais fatores apresentados, além da falta de medidas antimaláricas e campanhas de sensibilização socioambiental naquele momento.

Canelas et al. (2019) classifica a incidência parasitária anual na Amazônia brasileira em 3 categorias, são elas: alta transmissão (API $\geq 50)$, moderada transmissão $(10 \leq$ API <50) e baixa transmissão $(1 \leq$ API $<10)$. Neste estudo, os valores de incidência oscilaram durante os 10 anos de estudo, apresentando picos máximos entre 2011 e 2014, com 142,7 e 104,3 respectivamente (Tabela 1).

Tabela 1 - Análise da incidência parasitária anual no município de Cametá- PA.

\begin{tabular}{cc}
\hline Ano de ocorrência & API $^{*}$ \\
\hline 2008 & 0,14 \\
2009 & 0,23 \\
2010 & 28,65 \\
2011 & 142,74 \\
2012 & 23,79 \\
2013 & 1,86 \\
2014 & 104,30 \\
2015 & 0,02 \\
2016 & 0,04 \\
2017 & 10,15 \\
2018 & 82,38
\end{tabular}

*Incidência parasitária anual

Fonte: Dados do estudo, 2019. 
Segundo Canelas et al. (2019), durante os anos de 2010 a 2013 o município de Cametá esteve classificado como uma área de transmissão moderada ( $10 \leq$ API $<50)$, mas a partir de 2014 o município enquadrou-se em uma área de baixa transmissão ( 1 < API < 10), porém, no presente estudo foi possível verificar que específicamente no ano de 2014 o API atingiu o valor de 104,3, o que segundo a classificação demostra ser de alta transmissão (API $\geq 50$ ). Outro destaque é sobre a incidência parasitária anual, que apresenta aumento gradual em 2017 e 2018, com 10,1 e 82,4, respectivamente.

Braz e Barcellos (2018) ao analisar o processo de eliminação da transmissão da malária na Amazônia brasileira utilizando uma abordagem espacial da variação da incidência da doença no ano de 2016, o município de Cametá encontra-se em uma região classificada como G3C (em busca da redução, com epidemia), contrapondo-se com o presente estudo, no qual ao encontrar 6 casos de Malária, obtem a incidência parasitária anual de 0,04 casos nesse ano. Esse resultado possivelmente está relacionado a possíveis falhas de notificação no sistema de informação SIVEP-Malária, que acometem importantes dados de conhecimento público de saúde para o ano de 2016.

Observa-se na figura 3 a distribuição das infecções de malária segundo o sexo, havendo predominância no sexo masculino $(59,4 \%)$ em relação ao feminino (40,6\%). Esse mesmo padrão também se apresenta em outros estudos como Parise (2014), Couto et al. (2010) e Monteiro et al. (2013), com a maior ocorrência da malária está entre indivíduos do sexo masculino. Segundo Recht et al. (2017), jovens do sexo masculino apresentam maior vulnerabilidade, principalmente, devido as atividades realizadas, como, por exemplo, trabalho de mineração, que os expõem a picadas dos vetores.

Na variável espécie do parasito, Plasmodium vivax foi predominante com 99,7\% (34.394 agravos), seguido por P. falciparum com 0,25\% (92 agravos) e infecções mistas 0,05\% (16 agravos) dos casos de infecções em todo a série temporal. Como encontrado neste estudo, Dombrowski et al. (2018) encontrou, no município de Cruzeiro do Sul no estado do Acre, o P. vivax como Plasmodium predominante, sendo o mesmo padrão encontrado também por Arruda et al. (2016) no mesmo estado. A predominância dessa espécie está em vários países que compõem parte da flora amazônica, como analisado por Recht et al. (2017). 
Figura 3. Distribuição por sexo do número de casos positivos de Malária, 2008 - 2018.

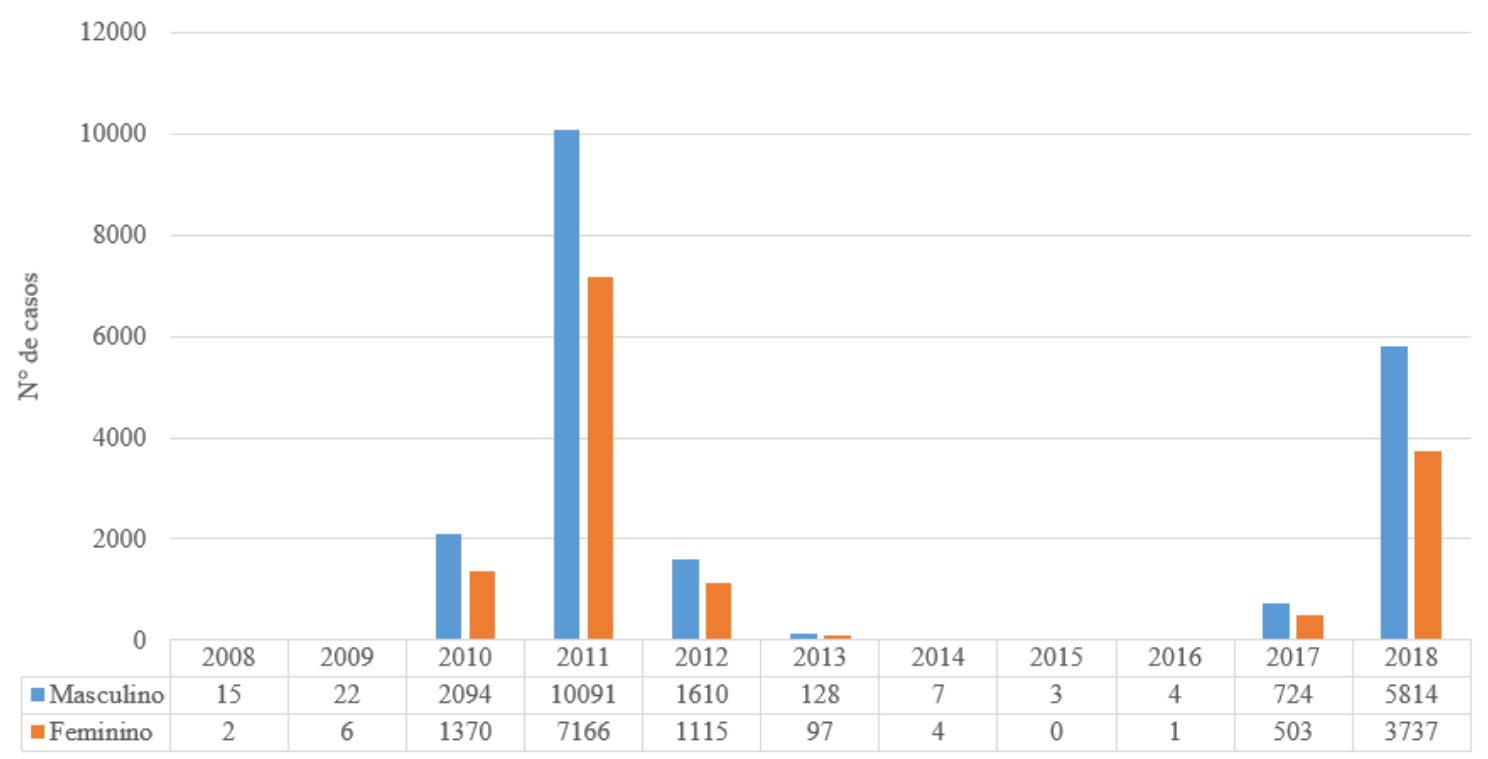

Fonte: SIVEP-Malária, 2019.

Embora o número de casos com o Pasmodium falciparum, neste estudo, seja inferior ao do P. vivax, o primeiro é o parasita da malária de maior patogenicidade ao ser humano, devido à magnitude das mortes atribuíveis à espécie. No entanto, cientistas no mundo todo observam alta prevalência de infecções sub-microscópicas por $P$. falciparum entre residentes assintomáticos (ELBADRY et al., 2015, TIEDJE et al., 2017). A Organização Pan-Americana de Saúde (2016) alerta que ambas as espécies $P$. falciparum e P. vivax são as que apresentam maior ameaça à saúde, pois estão associados a internações e óbitos (LAPOUBLE et al., 2015). Calculase que seis a oito milhões de infecções e 80.000 mortes ocorram todos os anos por conta desta endemia (SILVA; PAIVA, 2015, PINA et al., 2014).

Na figura 4 é apresentado o número de infecções por malária segundo a faixa etária, onde o público entre 20 a 29 anos apresentou maior vulnerabilidade frente a doença, com queda brusca no número de casos conforme o aumento da faixa de idade. A malária apresenta uma ampla distribuição em todos os segmentos etários, atingindo os extremos da vida, mas principalmente as faixas etárias ativas, de média idade (ex. Sousa et al., 2015; silva et al., 2017; Hermes et al., 2013). Apesar do maior número de casos nas faixas etárias ativas, tanto nos estudos de Sousa et al. 2015 quando este, também se encontra um grande acometimento em faixas etárias mais jovens, evidenciadas nesta pesquisa com a incidência de $31 \%$ em crianças com menos de 14 anos de idade. 
Figura 4. Número de casos agravos segundo a faixa etária no período estudado no município de Cametá- PA.

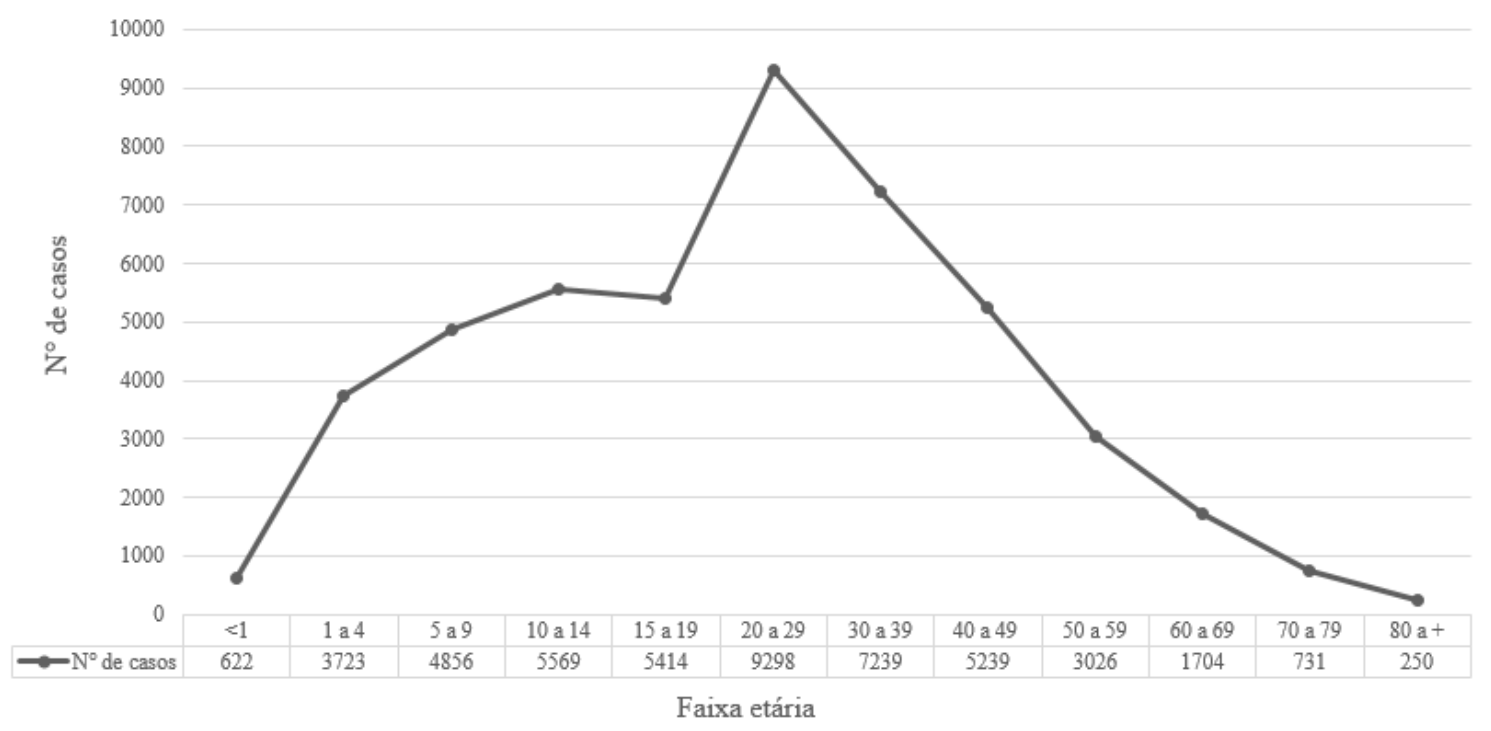

Fonte: SIVEP-Malária, 2018.

Com técnicas de análise espacial, foi possível observar que a distribuição espacial das infecções ao longo dos 11 anos no município de Cametá ocorreram tanto na zona rural como na zona urbana (Figura 5).

Figura 5. Áreas de provável infecção na zona rural e urbana do municipio de Cametá- PA.



Fonte: SIVEP-Malária e Google Earth, 2019. 
Couto et al. (2010) ao avaliar a malária no estado de São Paulo, verificou a associação da malária com várias ocupações sociais, principalmente lavrador e lazer, as quais estão ligadas diretamente a zona rural, como, por exemplo, atividade de sustento e ecoturismo, respectivamente. Apesar do caráter rural da doença, atualmente o percentual tem aumentado em espaços urbanos (LAPOUBLE; SANTELLI; MUNIZ-JUNQUEIRA, 2015; BRASIL, 2015). Ramalho et al. (2018) relata que no município de Porto Velho, capital do Estado de Rondônia, 38,82\% dos indivíduos apontaram a zona urbana como possível área de infecção. Dessa maneira, observa-se que cada vez mais a malária está obtendo um caráter urbano, pressupondo que haja uma falha no plano de erradicação da doença.

A malária urbana e peri-urbana, em oposição à malária exclusivamente rural, em locais pobres e isolados, é agora um fenômeno reconhecido, devido em parte às viagens, mais precisamente à movimentos populacionais maiores, especialmente de áreas rurais às urbanas e peri-urbanas (RECHT et al., 2017). Em Cametá é possível observar (Figura 6) que todas as áreas urbanas do município são áreas prováveis de infecção.

Figura 6. Áreas da provável infecção na zona urbana do municipio de Cametá- PA.

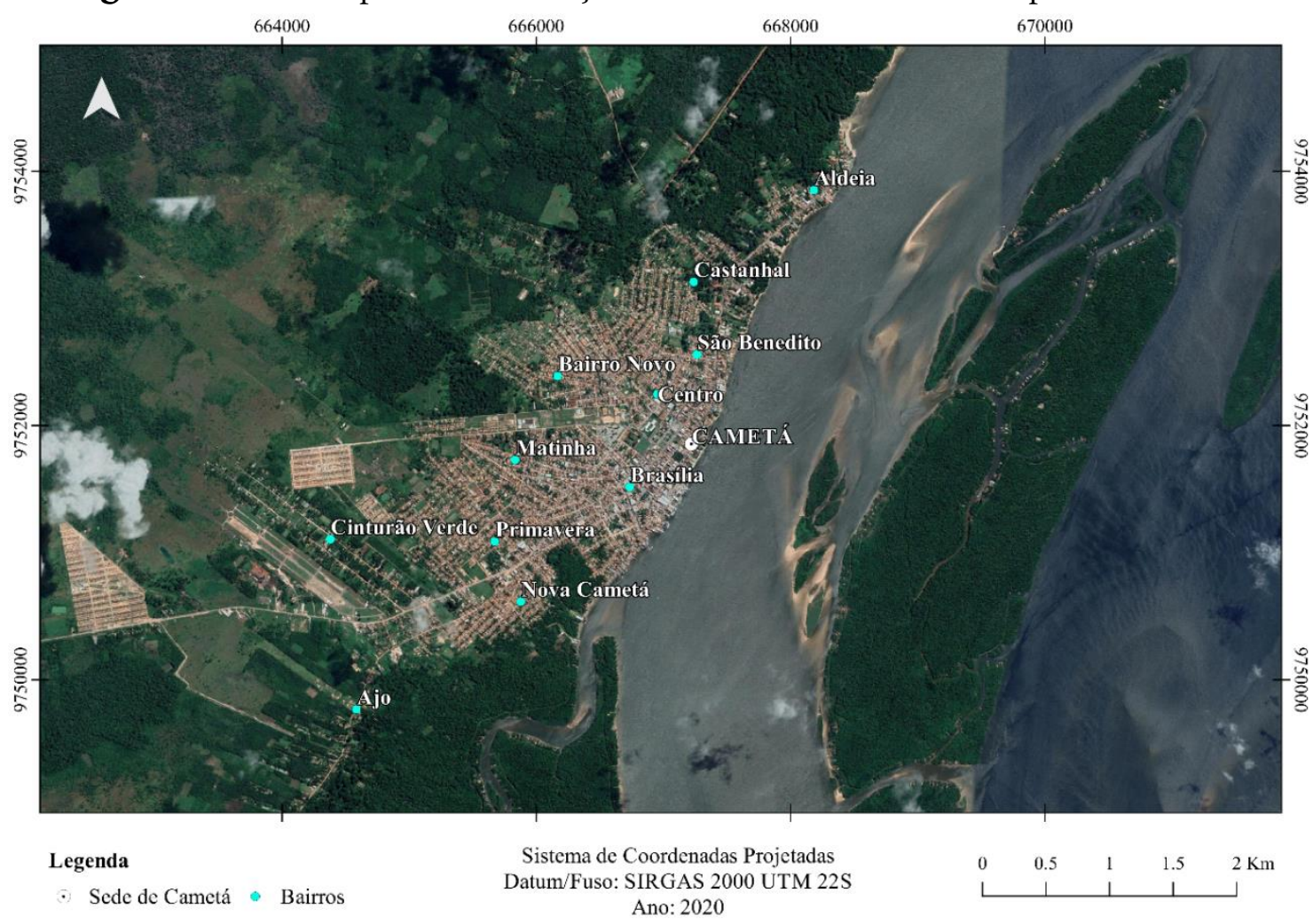


Fonte: SIVEP-Malária e Google Earth, 2019.

Como há uma diversidade mosquitos Anopheles que são responsáveis pela propagação da malária para os seres humanos (MASSEY et al., 2016), há necessidade de estudos com viés eco-epidemiológico de Anopheles em áreas de transmissão, pois os mecanismos de adaptação dos vetores podem estar associados aos eventos da infecção em áreas urbanas, incluindo este município de estudo, Cametá. A biologia de cada espécie de vetor define características importantes para o estudo da epidemiologia da malária, já que algumas espécies de Anopheles preferem áreas florestais e outras estão mais associadas a áreas desmatadas (MASELLI et al., 2014, LAPORTA et al., 2015), por exemplo.

Atualmente, existem programas de controle que visam maximizar a redução de casos e morte por malária. Essas estratégias estão entre controle de vetores, quimioprevenção, testes diagnósticos e tratamentos de casos confirmados da infecção, para que então possa ter uma melhor ação nos cuidados e tratamentos, além da prevenção dos possíveis focos de transmissão (WHO, 2018). Entretanto, como observado neste estudo, as estratégias de controle da malária ainda não foram suficientes para erradicar surtos da infecção no país, principalmente se tratando da região amazônica brasileira, pois a correlação de fatores ambientais aliados às ações antrópicas surte efeitos negativos na proliferação dos mosquitos transmissores da malária (LAPOUBLE et al., 2015), resultando em picos de casos.

Assim, na região amazônica, a propagação da malária é tida como cenário difícil e desafiador, pois a baixa densidade populacional e a falta de rotas de transporte dificultam operacionalmente a prestação e manutenção de cuidados de saúde e medidas preventivas, em especial em localidades mais distantes como espaços rurais (OLIVEIRA et al., 2010). Em vista disso, é de suma importância a descentralização do sistema de saúde, para que as medidas de tratamento, principalmente os medicamentos antimaláricos estejam ao alcance dos indivíduos que vivem em áreas de risco da infecção. 


\section{CONSIDERAÇÕES FINAIS}

O banco de dados identificou um pico da infecção no ano de 2011, mas com uma diminuição nos anos seguintes, com retorno de novo pico de infecção em 2014 e aumentando progressivamente o número de infecções a partir de 2017, chegando 68,4\% de aumento em 2018, em relação ao ano de 2017. Isso mostra que a incidência na cidade de Cametá oscilou durante os 10 anos de estudo.

Identificou-se que a espécie Plasmodium vivax foi predominante nas infecções, seguido por P. falciparum, tendo infecções mistas em poucos agravos.

Foi possível verificar que a malária esteve presente tanto na zona rural quanto na zona urbana do município, desse modo caracterizando uma participação efetiva da infecção em ambos os ambientes. Assim, é importante que a secretaria de vigilância epidemiológica do município adote medidas de prevenção e combate em ambas as áreas, principalmente, pela progressão do retorno das infecções no ano de 2018.

\section{REFERÊNCIAS}

ARRUDA, E. F.; ARAUjO, F. M.; GUIMARÃES, M. G. S.; NOGUEIRA, R.; RAMALHO, A. A.; SILVA-NUNES, M. Associação entre malária e anemia em área urbana de transmissão do Plasmodium: Mâncio Lima, Acre, Brasil. Cad. Saúde Pública, n³2, v. 9, 2016. Acesso em: 29 mar. 2019.

BRASIL. Boletim Epidemiológico. Secretaria de Vigilância em Saúde - Ministério da Saúde, nº 43, v. 46, 2015. ISSN: 2358 9450. Disponível em: http://portalarquivos2.saude.gov.br/images/pdf/2015/dezembro/16/2015003---Mal--ria.pdf. Acesso em: 28 mar. 2019.

BRASIL. Boletim epidemiológico: malária. Ministério da saúde - Sistema de vigilância sanitária. 2018. Disponível em: http://www.parauapebas.pa.gov.br/index.php/component/k2/item/download/103_2boof958e0c0e455ece713 08d2a36f48.html. Acesso em: 28 mar. 2019.

BRASIL. Brasil tem o menor número de casos de malária dos últimos 35 anos. 2017. Disponível em: http://www.brasil.gov.br/noticias/saude/2016/04/brasil-tem-o-menor-numero-de-casos-de-malaria-dosultimos-35-anos. Acesso em: 29 mar. 2019.

BRASIL. Plano de eliminação de malária no Brasil. 2016. Disponível em: http://portalarquivos2.saude.gov.br/images/pdf/2017/janeiro/04/Plano-eliminacao-malaria-pub.pdf. Acesso em: 29 mar. 2019.

BRAZ, R. M.; BARCELLOS, C. Análise do processo de eliminação da transmissão da malária na Amazônia brasileira com abordagem espacial da variação da incidência da doença em 2016. Epidemiol. Serv. Saúde, nº 27, v. 3, 2018. Disponível em: https://doi.org/10.5123/S1679-49742018000300010. Acesso em: 29 mar. 2019. 
BRAZ, R. M.; BARCELLOS, C. Analysis of the process of malaria transmission elimination with a spatial approach to incidence variation in the Brazilian Amazon, 2016. Epidemiol. Serv. Saude, n. 27, v. 3, 2018. Disponível em: http://www.scielo.br/pdf/ress/v27n3/2237-9622-ress-27-03-e2017253.pdf. Acesso em: 31 jul. 2019.

CANELAS, T.; CASTILLO-SALGADO, C.; BAQUERO, O. S.; RIBEIRO, H. Environmental and socioeconomic analysis of malaria transmission in the Brazilian Amazon, 2010-2015. Rer. Saúde Pública, v. 53, 2019. Disponível em: http://www.scielo.br/scielo.php?pid=S0034-89102019000100244\&script=sci_arttext. Acesso em: 31 jul. 2019.

CHIARAVALLOTI-NETO, F. O geoprocessamento e saúde pública. Arq. Ciênc. Saúde, nº 23, v. 4, 2016. Disponível em: https://doi.org/10.17696/2318-3691.23.4.2016.661. Acesso em: 28 mar. 2019.

COSNER, C.; BEIER, J. C.; CANTRELL, R. S.; IMPOINVIL, D.; KAPITANSKI, L.; POTTS, M. D.; TROYO, A.; RUAN, S. The effects of human movement on the persistence of vector-borne diseases. Journal of Theoretical Biology, v. 258, n. 4, 2009. Disponível em: https://www.ncbi.nlm.nih.gov/pmc/articles/PMC2684576/. Acesso em: 25 jun. 2019.

COUTO, R. D.; LATORRE, M. R. D. O.; SANTI, S. M. D.; NATAL, D. Malária autóctone notificada no Estado de São Paulo: aspectos clínicos e epidemiológicos de 1980 a 2007. Revista da Sociedade Brasileira de Medicina Tropical, $\quad$ v. $43, \quad 2010 . \quad$ Disponível em: https://www.researchgate.net/profile/Silvia_Di_Santi/publication/245848997_Malaria_autoctone_notificada _no_Estado_de_Sao_Paulo_aspectos_clinicos_e_epidemiologicos_de_1980_a_2007/links/53f635e90cf2fceacc70 c936.pdf. Acesso em: 25 jun. 2019.

ELBADRY, M.A., AL-KHEDERY, B., TAGLIAMONTE, M.S., YOWELL, C.A., RACCURT, C.P., EXISTE, A., BONCY, J., WEPPELMANN, T.A., BEAU DE ROCHARS, V.E.M., LEMOINE, J.F., OKECH, B. A.; DAME, J.B. High prevalence of asymptomatic malaria infections: a cross-sectional study in rural areas in six departments in Haiti. Malar J. 2015. Disponível em: https://malariajournal.biomedcentral.com/track/pdf/10.1186/s12936-015-1051-2. Acesso em: 29 mar. 2019.

ESTIMATIVA POPULACIONAL 2019. Instituto Brasileiro de Geografia e Estatística (IBGE). Acesso em: 07 jan. 2020.

Fundação Oswaldo Cruz - FIOCRUZ. Conheça a malária. 2015. Disponível em: http://www.fiocruz.br/ioc/media/malaria\%20folder.pdf. Acesso em:17 fev de 2018.

HERMES, S. C. N. M.; NUNES, V. L. B.; DORVAL, M. E. C.; BRILHANTE, A, F. Aspectos epidemiológicos da malária humana no município de Aripuanã, estado de Mato Grosso, Brasil, 2005 a 2010. Revista Brasileira de Geografia Médica e da Saúde, n. 9, v. 17, 2013. Disponível em: http://www.seer.ufu.br/index.php/hygeia/article/view/22717/13621. Acesso em: 25 jun. 2019.

LAPORTA, G. Z.; BURATTINI, M. N.; LEVY, D.; FUKUYA, L.A.; OLIVEIRA, T. M. P.; MASELLI, L. M. F.; CONN, J. E.; MASSAD, E.; BYDLOWSKI, S. P.; SALLUM, M. A. Plasmodium falciparum in the southeastern Atlantic forest: a challenge to the bromeliad-malaria paradigm? Malar J. Disponível em: https://www.ncbi.nlm.nih.gov/pubmed/25909655. Acesso em: 29 mar. 2019.

LAPOUBLE, O. M. M.; SANTELLI, A. C. F. S.; MUNIZ-JUNQUEIRA, M. I. Situação epidemiológica da malária na região amazônica brasileira, 2003 a 2012. Rev Panam Salud Publica, $\mathrm{n}^{\circ}$ 38, v. 4, 2015. Disponível em: https://www.scielosp.org/article/ssm/content/raw/?resource_ssm_path=/media/assets/rpsp/v38n4/v38n4a 06.pdf. Acesso em: 28 mar. 2019.

LAPOUBLE, O. M. M.; SANTELLI, A. C. F. S.; MUNIZ-JUNQUEIRA, M. I. Situação epidemiológica da malária na região amazônica brasileira, 2003 a 2012. Revista Panamericana de Salud Pública. 2015. Disponível em: https://www.scielosp.org/article/rpsp/2015.v38n4/300-306/. Acesso em: 31 jul. 019.

MACIEL, G. B. M. L.; ESPINOSA, M. M.; ATANAKA-SANTOS, M. Epidemiologia da malária no município de Colniza, Estado de Mato Grosso, Brasil: estudo descritivo do período de 2003 a 2009. Epidemiol. Serv. Saúde, v. 22, n. 3, 2013. Disponível em: http://scielo.iec.gov.br/pdf/ess/v22n3/v22n3a11.pdf. Acesso em: 25 jun. 2019. 
MASELLI, L.M.F., LEVY, D., LAPORTA, G.Z., MONTEIRO, A.M., FUKUYA, L.A., FERREIRA-DA-CRUZ, M.F., DANIELRIBEIRO, C.T., DORLHIACLLACER, P.E., SALLUM, M.A.M. \& BYDLOWSKI, S.P. Detection of Plasmodium falciparum and Plasmodium vivax subclinical infection in nonendemic region: implications for blood transfusion and malaria epidemiology. Malar J. 2014. Disponível em: https://www.ncbi.nlm.nih.gov/pubmed/24906577. Acesso em: 29 mar. 2019.

MASSEY, N. C.; GARROD, G.; WIEBE, A.; HENRY, A. J.; HUANG, Z.; MOYES, C. L.; SINKA, M. E. A global bionomic database for the dominant vectors of human malaria. Sci. Data, v. 6, 2016. Disponível em: https://www.ncbi.nlm.nih.gov/pubmed/26927852. Acesso em: 29 mar. 2019.

MONTEIRO, M. R. C. C.; RIBEIRO, M. C.; FERNANDES, S. C. Aspectos clínicos e epidemiológicos da malária em um hospital universitário de Belém, Estado do Pará, Brasil. Rev Pan-Amaz Saude, v. 4, n. 2, 2013. Disponível em: http://scielo.iec.gov.br/pdf/rpas/v4n2/v4n2a05.pdf. Acesso em: 25 jun. 2019.

OLIVEIRA, F. J.; LACERDA M. V. G.; BRASIL, P.; LADISLAU, J. L. B.; TAUIL, P. L.; DANIEL-RIBEIRO, C. T. Malária no Brasil: uma visão geral. BioMed Central, nº 9, v. 115, 2010.

Organização Pan-Americana de Saúde - OPAS. Malária. 2016. Disponível em: https://www.paho.org/bra/index.php?option=com_content\&view=article\&id=5287:malaria-2\&Itemid=875.

Acesso em: 28 mar. 2019.

PINA, C. A.; BRASIL, P.; SANTI, S. M.; ARAUJO, M. P.; SUÁREZ-MUTIS, M. C.; SANTELLI, A. C. F. S.; OLIVEIRAFERREIRA, J.; LOURENÇO-DE-OLIVEIRA, R.; DANIEL-RIBEIRO, C. T. Malaria in Brazil: what happens outside the Amazonian endemic region. Mem. Inst. Oswaldo Cruz, n 5, v. 109, 2014.

RAMALHO, A. L. S.; CASTRO, O. B.; LIMA, R. A.; DENE, Y.; LEITE, G. L.; SOUZA, A. R.; CARVALHO, L. H.; DINIZ, S. C.; OLIVEIRA, M. G.; PEREIRA, D. B. Uso de terapias naturais durante o tratamento da infecção de plasmodium vivax no município de Porto Velho-RO. Revista Eletrônica Acervo Saúde, v. 13, 2018. ISSN 2178-2091. Disponível em: https://www.acervosaude.com.br/doc/REAS390.pdf. Acesso em: 28 mar. 2019.

RECHT, J.; SIQUEIRA, A. M.; MONTEIRO, W. M.; HERRERA, S. M.; HERRERA, S.; LACERDA, M. V. G. Malaria in Brazil, Colombia, Peru and Venezuela: current challengs in malaria control and elimination. Malar j. v. 16, n. 1, p. 273, 2017.

RECHT, J.; SIQUEIRA, A. M.; MONTEIRO, W. M.; HERRERA, S. M.; HERRERA, S.; MARCUS V. G. LACERDA, M. V. G. Malaria in Brazil, Colombia, Peru and Venezuela: current challenges in malária control and elimination. Malar J. v. 16, n. 273, 2017. Disponível em: https://www.ncbi.nlm.nih.gov/pubmed/28676055. Acesso em: 25 jun. 2019.

ROGERSON, S. J.; DESAI, M.; MAYOR, A.; SICURI, E.; TAYLOR, S. M.; VAN EIJK, A.M. Burden, pathology, and costs of malaria in pregnancy: new developments for an old problem. Lancet Infect Dis. V. 3, n. 12, p. 08-20, 2018.

ROMI, R.; BOCCOLINI, D. VALLORANI, R.; SEVERINI, F.; TOMA, L.; COCCHI, M.; TAMBURRO, A.; MESSERI, G.; CRISCI, A.; ANGELI, L.; COSTANTINI, R.; RAFAELLI, I.; PONTUALE, G.; THJERY, I.; LANDIER, A.; GOFF, G. L.; FAUSTO, A. M.; DI LUCA, M. Assessment of the ris of malária re-introduction in the Maremma (Central Italy) using a multifactorial approach. Malar J. v. 11, n. 98, 2012. Disponível em: https://malariajournal.biomedcentral.com/articles/10.1186/1475-2875-11-98. Acesso em: 25 jun. 2019.

SANTOS, V. R.; YOKOO, E. M.; SOUZA-SANTOS, R.; ATANAKA-SANTOS, M. Fatores socioambientais associados à distribuição espacial de malária no assentamento Vale do Amanhecer, Município de Juruena, Estado de Mato Grosso, 2005. Revista da Sociedade Brasileira de Medicina Tropical, v. 42, 2009. Disponível em: http://www.scielo.br/scielo.php?pid=S0037-86822009000100010\&script=sci_abstract\&tlng=pt. Acesso em: 25 jun. 2019.

SILVA, R.; PAIVA, C. H. A. The Juscelino Kubitschek government and the Brazilian Malaria Control and Eradication Working Group: collaboration and conflicts in Brazilian and international health agenda, 1958-1961. Hist. Cienc. Saude-Manguinhos, n 1, v. 22, 2015. 
SILVA, Y. F.; GUTJAHR, A. L. N.; BRAGA, C. E. S.; MARQUES, J. R. A. Casos de malária registrados no município de Cândido Mendes, Maranhão, no período de 2009-2014. Enciclopédia biosfera, Centro Científico Conhecer, v. 14, n. 25, 2017. Disponível em: http://www.conhecer.org.br/enciclop/2017a/sau/casos\%20de\%20malaria.pdf. Acesso em: 25 jun. 2019.

SIQUEIRA, A. M.; MESONES-LAPOUBLE, O.; MARCHESINI, P.; SAMPAIO, V. S.; BRASIL, P.; TAUIL, P. L.; FONTES, C. J.; COSTA, F. T. M. DANIEL- RIBEIRO, C. T.; LACERDA, M. V. G.; DAMASCENO, C. P.; SANTELLI, A. C. S. Plasmodium vivax Landscape in Brazil: Scenario and Challenges. The American Journal of Tropical Medicine and Hygiene. 2016. Disponível em: https://doi.org/10.4269/ajtmh.16-0204. Acesso em: 28 mar. 2019.

SOUSA, J. R.; SANTOS, A. C. F.; ALMEIDA, W. S.; ALBARADO, K. V. P.; MAGNO, L. D.; ROCHA, J. A. M.; PIMENTEL, Z. N. S. Situação da malária na região do Baixo Amazonas, estado do Pará, Brasil, de 2009 a 2013: um enfoque epidemiológico. Rev. Pan-Amaz Saude, v. 6, n. 4, p 39 - 47, 2015. Disponível em: http://scielo.iec.gov.br/pdf/rpas/v6n4/v6n4a06.pdf. Acesso em: 25 jun. 2019.

TIEDJE, K.E.; ODURO, A.; AGONGO, G.; ANYORIGIYA, T.; AZONGO, D.; AWINE, T.; GHANSAH, A.; PASCUAL, M.; KORAM, K.; DAY, K. P. Seasonal variation in the epidemiology of asymptomatic Plasmodium falciparum infections across two catchment areas in Bongo District, Ghana. Am. J. Trop. Med. Hyg., n 97, v. 1, 2017. Disponível em: https://doi.org/10.4269/ajtmh.16-0959. Acesso em: 29 mar. 2019.

VITOR-SILVA, S.; SIQUEIRA, A. M.; SAMPAIO, V. S.; GUINOVART, C.; REYES-LECCA, R. C.; MELO, G. C.; MONTEIRO, W. M.; PORTILLO, H. A.; ALONSO, P.; BASSAT, Q.; LACERDA, M. V. G. Declining malaria trasmission in rural Amazon: changing epidemiology and challenges to achieve elimination. Malaria Journal. 2016. Disponível em: https://malariajournal.biomedcentral.com/articles/10.1186/s12936-016-1326-2. Acesso em: 29 mar. 2019.

WOLFARTH-Couto, B.; SILVA, R. A. da; FILIZOLA, N. Variabilidade dos casos de malária e sua relação com a precipitação e nível d'água dos rios no Estado do Amazonas, Brasil. Cadernos de Saúde Pública, v. 35, n. 2, 2019.

WHO- World Health Organization. Overview of malaria elimination. 2018. Disponivel em: https://www.who.int/malaria/areas/elimination/overview/en/. Acesso em: 29 mar. 2019.

WHO- World Health Organization . World malaria report. 2016. Disponível em: https://apps.who.int/iris/bitstream/handle/10665/252038/9789241511711eng.pdf;jsessionid=EA4186333702CDFB4C1BF94FA851540E? sequence=1. Acesso em: 29 mar. 2019.

\section{(c) EY}

Este trabalho está licenciado com uma Licença Creative Commons - Atribuição 4.0 Internacional. 